\title{
P wave duration and morphology in patients with atrial fibrillation
}

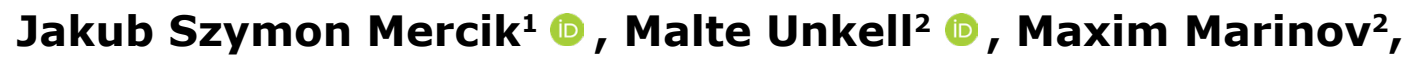 \\ Peter Stephan Wolff $\mathbb{D}^{\mathbb{D}}$, Jadwiga Radziejewska³, Jacek Gajek ${ }^{4}$
}

\author{
${ }^{1}$ Department of Emergency Medicine, Wrocław Medical University, Wrocław, Poland \\ ${ }^{2}$ Students' Scientific Association, Department of Emergency Medical Service, Wrocław Medical University, Wrocław, Poland \\ ${ }^{3}$ Kłodzko County Hospital, Kłodzko, Poland \\ ${ }^{4}$ Department of Emergency Medical Service, Wrocław Medical University, Wrocław, Poland
}

\begin{abstract}
Background: Functional and structural atrial changes contribute to AF. It decreases conduction velocity and forms intra atrial blocks. In the ECG those changes are manifested by the duration and morphology of the $P$ wave. Material and methods: The study group consisted of 50 patients with atrial fibrillation. There were 27 women and 23 men, aged $65.3+/-9.8$ years. 22 patients had paroxysmal AF and 28 had persistent AF, in the latter direct current cardio version was performed. Results: In patients with a prolonged episodes of atrial fibrillation the $P$ wave duration was longer in comparison to patients with sinus rhythm $(187.1+/-31.5$ vs 161.1 $+/-18.8 \mathrm{~ms} ; \mathrm{p}=0.006)$. There were significant differences in $\mathrm{P}$ wave duration among the patients with normal and abnormal interatrial conduction, with the longest duration in complete Bachmann's bundle block group $(152.7+/-17.5$ vs $165.3+/-15.3$ vs $207.9+/-27.5 \mathrm{~ms} ; \mathrm{p}<0.001)$. Conclusions: In patients with persistent atrial fibrillation the duration of the $\mathrm{P}$ wave is prolonged in comparison to paroxysmal. In the majority of patients prolongation of the $\mathrm{P}$ wave duration is dependent on different forms of conduction block. The morphological changes of $\mathrm{P}$ waves are caused by the arrhythmia rather than left atrial hypertrophy.
\end{abstract}

Keywords: atrial fibrillation - P wave duration - P wave morphology - Bachmann's bundle

\section{Citation}

Mercik JS, Unkell M, Marinov M, Wolff PS, Radziejewska J, Gajek J. P wave duration and morphology in patients with atrial fibrillation. Eur J Transl Clin Med. 2020;3(2):22-28.

DOI: $10.31373 /$ ejtcm/127800

Corresponding author:

Jakub Szymon Mercik, Department of Emergency Medicine, Wrocław Medical University, Wrocław, Poland

e-mail: jakub.mercik@wp.pl

No external funds.

Available online: www.ejtcm.gumed.edu.pl

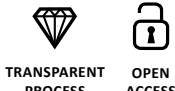

Copyright $®$ Medical University of Gdańsk

This is Open Access article distributed under the terms of the Creative Commons Attribution-ShareAlike 4.0 International. 


\section{Introduction}

Functional and structural changes in an atrial muscle constitute a substrate for an atrial fibrillation (AF) [1]. These changes are the result of numerous diseases which mainly include hypertension, ischemic heart disease, further heart defects, and in particular a mitral valve disease, diabetes, myocarditis, heart failure or, less commonly, hyperthyroidism [2]. Despite the fact that we consider the atria as a whole, AF is mainly caused by pathologies that negatively influence the left atrium. The pathophysiology of a structural damage to the atrial musculature consists mainly in stretching its cavities, followed by necrosis and/or apoptosis of cardiomyocytes and replacing them by connective tissue cells [3-4]. This slows down the global conduction velocity of the activation wave, leading to slow conduction zones and focal conduction block, and contributing to the formation of re-entry circuits, which promote arrhythmias. The impact of the described phenomena on the isolation of junctional areas between the left atrium and pulmonary veins, ultimately with the formation of trigger zones in this area, proves to be very significant [5-6].

The structural changes described above contribute to the formation of arrhythmogenic foci, perpetuating premature beats, and to atrial rhythms. Rapid arrhythmias induce local changes in the refractory period of the atrial muscle, which, combined with the structural changes, increase the possibility of re-entrant rhythms and leads to the persistence of AF [7]. The arrhythmic paroxysms contribute to further enlargement of the left atrium by increasing left ventricle filling pressure, which then leads to consecutive structural changes.

As a result of the above-described processes, in the electrocardiogram (ECG) we can observe changes in the duration and amplitude of the $P$ wave. Slow conduction and an enlargement of the left atrium are the causes of a prolonged $P$ wave duration [8-9]. The pressure overload, especially in the hypertension, may induce atrial muscle hypertrophy which might be displayed as an increased $P$ wave amplitude (an increase in the negative deflection in lead V1) [10]. However, a decrease in the number of cardiomyocytes and an increase in the content of connective tissue in the heart can lead to the opposite situation: a decrease in the $\mathrm{P}$ wave amplitude. Another issue is the appearance of intra- and interatrial conduction disturbances in the form of a partial or a complete Bachmann's bundle block. Changes in either the activation time or the direction of the conduction within the left atrium significantly affect the $P$ wave morphology in the case of a complete block [11]. Assessment of the latter parameter has not been extensively reported in the lite- rature so far. The purpose of our study is to assess the influence of the AF form on the duration and morphology of the $P$ wave in the electrocardiogram.

\section{Material and methods}

We recruited into this study patients who were treated for AF at a single cardiology department in the years 2012-2020. The inclusion criteria were: paroxysmal or persistent (including long-term persistent) AF and the patients' consent. The exclusion criteria were: permanent form of arrhythmia, low symptomatic AF, lack of patients' consent. We took detailed history regarding the antiarrhythmic drugs the patient was currently taking, including propafenone and amiodarone. Patients' co-morbidities were noted. As the precise duration of an arrhythmia episode was not possible to establish, we included the patients with persistent AF lasting from 4 to 24 weeks.

The $P$ wave duration and its morphology were analyzed using the LabSystem ${ }^{\mathrm{TM}}$ Pro EP Recording System (Boston Scientific, Marlborough, United States), where the ECG tracings allowed for assessing the sinus $P$ waves. The $P$ wave duration was measured in all leads at paper speed of $200 \mathrm{~mm} / \mathrm{s}$ and enhancement of 64-128. The $P$ wave morphology was assessed in lead II for the presence of notches (humps) and negative phases, indicating interatrial conduction disorders, known as Bachmann's bundle incomplete and complete conduction block. Another parameter was the amplitude of the $P$ wave. Additionally, in Bachmann's bundle incomplete block the separation of the right and left atrium $P$ waves peak was measured (if it exceeded $40 \mathrm{~ms}$ ). In lead V1, the amplitude of positive and negative phases was also measured. To avoid any influence of accidental measurement inaccuracies, all the measurements were repeated 5 times and the mean value was calculated.

In patients with a persistent AF, direct current cardioversion was performed under general anaesthesia under propofol $1 \mathrm{mg} / \mathrm{kg}$ and fentanyl $50 \mu \mathrm{g}$ administered intravenously. Single shock of $300 \mathrm{~J}$ was successful in all patients. The study was approved by the local Bioethical Committee at the Wrocław Medical University.

All continuous variables were presented as a mean and standard deviation. Comparisons were performed with the $U$ Mann-Whitney test for independent groups or Kruskal-Wallis ANOVA for multiple comparisons. Any dependent comparisons were performed using the Wilcoxon paired test. All categorical variables were presented as numbers and percentages. The comparisons were performed with the chi-square test. $P$ values $<0.05$ were considered statistically significant. 


\section{Results}

The study group consisted of 50 patients diagnosed with AF. There were 27 women and 23 men, aged $65,3+/-9,8$ years. There were 22 patients with paroxysmal AF (in sinus rhythm during examination) and 28 patients with persistent $A F$, in whom the direct current cardioversion was performed in order to restore sinus rhythm. The clinical characteristics of studied patients were presented in Table 1.

In patients with a prolonged episode of the $A F$, the $P$ wave duration was longer in comparison to patients with the sinus rhythm. The parameters and features of the $P$ waves were presented in Table 2 . The persistent AF group tended to have more pronounced interatrial conduction disorders but it did not reach statistical significance. In patients with the persistent $\mathrm{AF}$, the positive amplitude in lead V1 was significantly higher than in patients with the paroxysmal AF. All other parameters did not vary between the studied patients. The direct comparison of positive and negative deflection in lead V1 in both groups according to AF form discriminated the borderline difference in group with the persistent $A F(p=$ 0.061).

Table 2. $P$ wave parameters in the studied patients according to atrial fibrillation form

\begin{tabular}{|c|c|c|}
\hline $\begin{array}{c}\text { Patients with } \\
\text { paroxysmal atrial } \\
\text { fibrillation }\end{array}$ & $\begin{array}{c}\text { Patients with } \\
\text { persistent atrial } \\
\text { fibrillation }\end{array}$ & $\mathbf{P}$ \\
\hline 22 & 28 & - \\
\hline $161.1+/-18.8$ & $187.1+/-31.5$ & 0.006 \\
\hline 6 & 4 & 0.799 \\
\hline 11 & 14 & 0.532 \\
\hline 5 & 10 & 0.322 \\
\hline $56.5+/-23.3$ & $66.3+/-18.7$ & 0.354 \\
\hline $0.150+/-0.161$ & $0.109+/-0.037$ & 0.464 \\
\hline $0.055+/-0.023$ & $0.081+/-0.037$ & 0.005 \\
\hline $0.049+/-0.021$ & $0.061+/-0.036$ & 0.304 \\
\hline
\end{tabular}


The comparison of the presence of normal and abnormal Bachmann's bundle conduction did not yield any statistically significant result (16/22 vs. $24 / 28, p=0.91)$. The parameters of the $P$ waves presented according the interatrial conduction properties were presented in Table 3.

Significant differences in $\mathrm{P}$ wave duration were observed among the patients with a normal and an abnormal interatrial conduction, with the longest duration in the complete Bachmann's bundle block group. The differences in question inclined to be in concor- dance with an increasing age of the patients, even if the age of the patients, nor the other parameters (duration) were not statistically significant. While assessing the $\mathrm{P}$ wave morphology, an unexpected discovery was made. In 7 patients the features of the $P$ wave did not meet the criteria of a double humped $P$ wave as typically recognized in an incomplete Bachmann's block. We called them triple-humped $P$ waves, as they exhibited three distinct peaks in lead II. An example of such morphology was presented in Figure 1.

Table 3. $P$ wave parameters in all the studied patients according to interatrial conduction

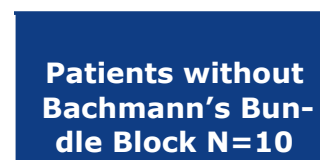

Patients with incomplete Bachmann's Bundle Block $\mathbf{N}=\mathbf{2 5}$

\section{Patients with complete Bachmann's Bundle Block $N=15$}

\begin{tabular}{|c|c|c|c|}
\hline Age (years) & $59.5+/-10.0$ & $65.1+/-11.4$ & 0.102 \\
\hline P wave duration (ms) & $152.7+/-17.5$ & $165.3+/-15.3$ & $<8.8+/-6.3$ \\
\hline Amplitude II lead (mV) & $0.118+/-0.031$ & $0.144+/-0.147$ & $0.106+/-0.065$ \\
\hline Amplitude V1 lead positive (mV) & $0.070+/-0.039$ & $0.070+/-0.037$ & $0.069+/-0.027$ \\
\hline Amplitude V1 lead negative (mv) & $0.054+/-0.027$ & $0.062+/-0.035$ & $0.046+/-0.023$ \\
\hline
\end{tabular}

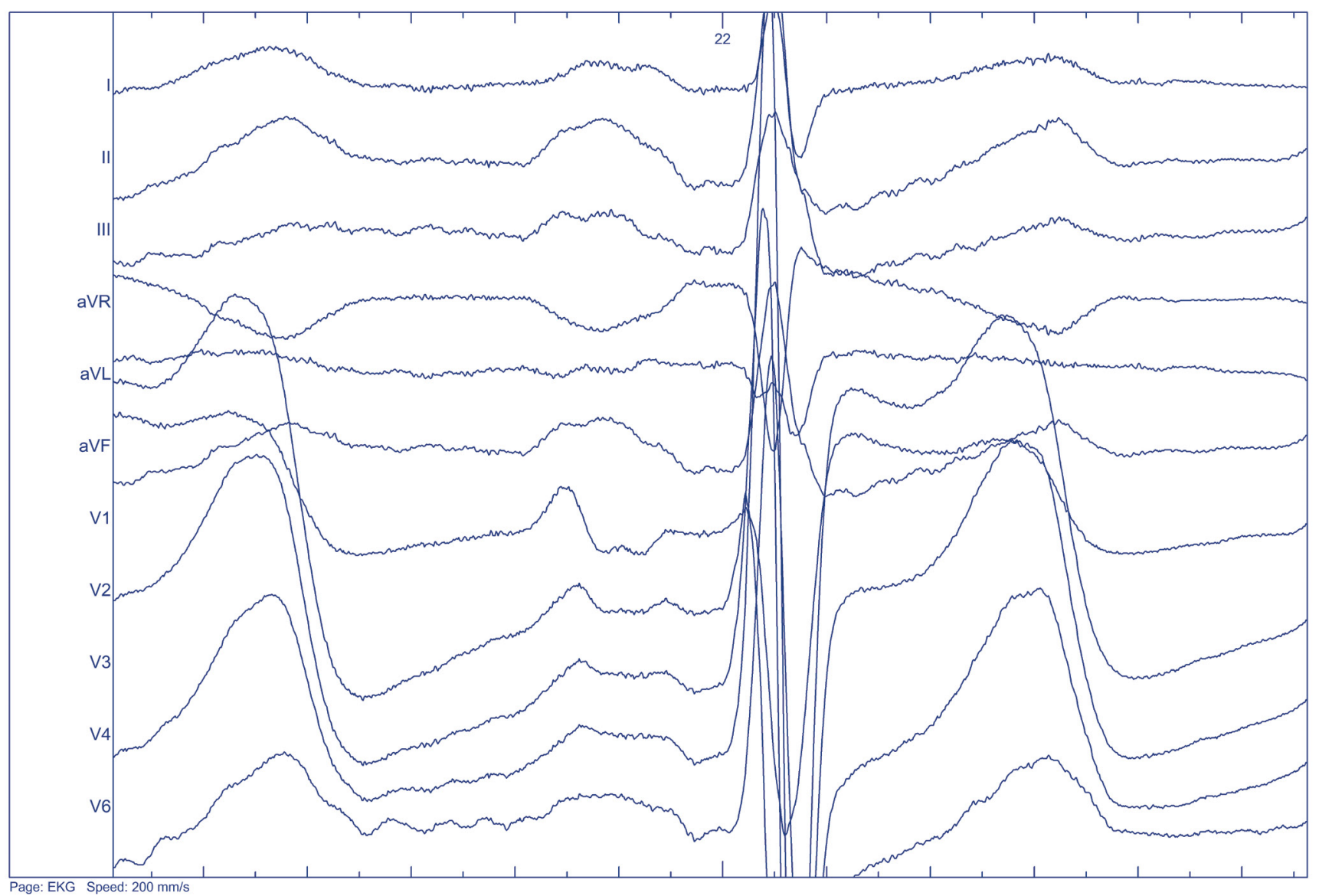

Figure 1. Triple humped $P$ wave. Note the 3 peaks in $P$ wave in lead II (present also in other leads). Paper speed 200 mm/s, magnification $64 x$. 
The comparison of the assessed parameters of the triple-humped $\mathrm{P}$ wave in the studied groups were shown in Table 4.

The mean duration of the triple-humped $\mathrm{P}$ waves from the persistent AF group emerged to be longer than the single-humped $P$ wave from the paroxysmal $A F$, but the number of patients did not allow the statistical analysis.

\section{Discussion}

Our key finding is the effect of an ongoing arrhythmia on the extension of the $P$ wave duration of the electrocardiogram, indicating AF-induced intra-atrial and inter-atrial conduction disorders and, most probably, atrial enlargement. The latter phenomenon is clearly associated with an increase in the left ventricular filling pressure and is probably dependent on the ventricular rate [12]. Data on the effect of AF on the muscle conduction is more scarce and equivocal, though.

Due to the fact that the subgroups of patients with paroxysmal and persistent AF were comparable in terms of age, gender distribution, comorbidities, and antiarrhythmic treatment, which might have affected the duration of the $P$ wave, it should be assumed that our observations are dependent only on the presence or absence of an ongoing arrhythmia [1]. This is in line with the clinical observation made years ago, contained in the in the term "AF begets AF" created by Wijffels et al. [13]. The electrical cardioversion itself had no effect on the duration of the $P$ wave in one study as the parameter was stable immediately after the procedure and 24 hours later [14].

It should be borne in mind that the precise methodology for measuring the duration of the $P$ wave adopted in our study is qualitatively different from the one that was used by other researchers [15-16]. It is not without reason that a few years ago we showed in a similar assessment the lack of $P$ wave dispersion, which is related to the inaccuracy of the measurement [17]. In fact, this approach exactly corresponds to Bayes de Luna's suggestions in the paper "Diagnosis of interatrial Block" only from 2017 where it was explicitly ordered to measure the $\mathrm{P}$ wave from its earliest beginning in any lead to the latest end in any lead [18].

Another conclusion from our research is the fact that there is a significant relationship between the duration of the $P$ wave and the separation of the right and left atrium peaks in lead II. Although the difference in the duration of this separation in both groups of patients turned out to be statistically insignificant and probably resulted from the percentage of patients with an incomplete conduction block in the Bachmann's bundle in both groups, it seems to be obvious that the extension of the $P$ wave peaks is mainly due to the prolongation of conduction in the bundle and not within the atrial muscle [19].

A further learning from our work is the increase in the amplitude of the initial phase of the $P$ wave in lead V1. This seems to reflect the known electrocardiographic relationship. Under normal conditions, the overlap of the left atrial negative phase with the right atrial positive phase causes a decrease in the former. Only a significant increase in the mass of the left atrium increasing the negative deflection in V1 results in the formation of a typical configuration - with a negative predominance in this lead [8]. Interestingly, our results point at another important relationship. In both examined groups, the positive deflection in lead V1 is greater than the negative deflection in this lead. It speaks strongly for the possibility that among the patients examined by us, it is interatrial conduction disturbances and not hypertrophy, e.g. hypertensive hypertrophy of

Table 4. Triple humped $P$ wave characteristics

\begin{tabular}{|c|c|}
\hline $\begin{array}{c}\text { Patients with } \\
\text { paroxysmal atrial fibrillation }\end{array}$ & $\begin{array}{c}\text { Patients with } \\
\text { persistent atrial fibrillation }\end{array}$ \\
\hline 1 & 6 \\
\hline 158.3 & $176.6+/-13.2$ \\
\hline 76 & $80.8+/-8.9$ \\
\hline 0.088 & $0.106+/-0.026$ \\
\hline 0.081 & $0.084+/-0.069$ \\
\hline 0.056 & $0.060+/-0.042$ \\
\hline
\end{tabular}


the left atrium as a cause of AF [4-5], that are prevailing.

When analyzing the entire group of patients, it should be assumed that the presence and the type of conduction disorders in the Bachmann's bundle are responsible for the increase of the duration of the $P$ wave. While there are no similar articles yet, and the only one referred to similar conclusions [20], this is consistent with the literature on Bachman's bundle conduction disorders [10-11, 19].

The analysis of the mean age in our group of patients without, with an incomplete, or with a full block of conduction in the Bachmann's bundle, even if not statistically significant, may indicate phases of dependence of the disease progression with age. The clear statistical relation could be expected with the increase of the number of patients. A more numerous group could make it possible to distinguish comparisons of patients with paroxysmal and persistent forms of the $A F$ in subgroups derived according to the interatrial conduction status.

The presence of the triple-humped $P$ waves have not been reported in the literature so far, even if the term 'thriphasic' (three-phased) was used in one animal experimental study by Pasławska et al. In the figure reported by them, the precise assessment shows a rather multiphasic pattern, as the initial phase is negative and the other two ones are positive [21]. In our small patients' series, the $\mathrm{P}$ wave morphology in lead II exhibits three more or less distinct positive peaks, indicating the activation of the left atrium through the Bachmann's bundle, with a kind of an additional conduction obstacle (compartmentation) in the left atrium. As we did not assess the anatomical and echocardiographic morphology of the atria, the final conclusion on the nature of the reported phenomenon remains uncertain and requires further research. Whether this observation has any clinical importance remains unclear and requires further study.

An important limitation of our study is a relatively small number of patients. As our assessment is based on a very precise measurement method using the electrophysiological recording system, we are aware that our results could not be directly comparable to the other authors' measurements. As we already mentioned, the duration of the persistent AF was not possible to be exactly established. Because we hypothesise that the extension of the $P$ wave duration is mainly caused by the arrhythmia itself, it should be understood as the main limitation of our research, even if the available data contradicts such a correlation [14]. The other important issue, that was not mentioned in the study, was the influence of a prolonged PR interval on the risk of AF development. The combination of atrial and atrioventricular abnormalities should constitute the objective of a further investigation.

\section{Conclusions}

In patients with the $A F$, the duration of the $P$ wave is prolonged. The persistent arrhythmia affects this parameter to a greater extent. In the majority of patients, the prolongation of the $\mathrm{P}$ wave duration is caused by different forms of the interatrial conduction block. The morphological changes of the $P$ waves indicate the causal relationship of the arrhythmia rather with interatrial conduction disorders than with a left atrial hypertrophy.

\section{References}

1. Allessie MA, Boyden PA, Camm AJ, Kléber AG, Lab MJ, Legato MJ, et al. Pathophysiology and Prevention of Atrial Fibrillation. Circulation [Internet]. 2001 Feb 6;103(5):769-77. Available from: https://doi.org/10.1161/01.CIR.103.5.769

2. Claeys MJ, Mullens W, Vandekerckhove Y, Duytschaever M, De Maeyer C, Pasquet A. Summary of 2016 ESC guidelines on heart failure, atrial fibrillation, dyslipidaemia and cardiovascular prevention. Acta Cardiol [Internet]. 2017 Nov 2;72(6):610-5. Available from: https://www.tandfonline.com/doi/full/10.1080/00015385.2017.1319681

3. Nattel S, Burstein B, Dobrev D. Atrial Remodeling and Atrial Fibrillation. Circ Arrhythmia Electrophysiol [Internet]. 2008 Apr;1(1):62-73. Available from: https://www.ahajournals.org/doi/10.1161/CIRCEP.107.754564

4. Schoonderwoerd BA, Van Gelder IC, Van Veldhuisen DJ, Van den Berg MP, Crijns HJGM. Electrical and Structural Remodeling: Role in the Genesis and Maintenance of Atrial Fibrillation. Prog Cardiovasc Dis [Internet]. 2005 Nov;48(3):153-68. Available from: http://www.sciencedirect.com/science/article/pii/S0033062005000782

5. Haïssaguerre M, Jaïs P, Shah DC, Takahashi A, Hocini M, Quiniou G, et al. Spontaneous Initiation of Atrial Fibrillation by Ectopic Beats Originating in the Pulmonary Veins. N Engl J Med [Internet]. 1998 Sep 3;339(10):659-66. Available from: https://doi.org/10.1056/NEJM199809033391003

6. Po SS, Li Y, Tang D, Liu H, Geng N, Jackman WM, et al. Rapid and Stable Re-Entry Within the Pulmonary Vein as a Mechanism Initiating Paroxysmal Atrial Fibrillation. J Am Coll Cardiol [Internet]. 2005 Jun 7;45(11):1871-7. Available from: https://doi.org/10.1016/i.jacc.2005.02.070 
7. Nitta T, Ishii Y, Miyagi Y, Ohmori H, Sakamoto S, Tanaka S. Concurrent multiple left atrial focal activations with fibrillatory conduction and right atrial focal or reentrant activation as the mechanism in atrial fibrillation. J Thorac Cardiovasc Surg [Internet]. 2004 Mar;127(3):770-8. Available from: http://www.sciencedirect.com/science/article/pii/ S002252230301403X

8. Chirife R, Feitosa GS, Frankl WS. Electrocardiographic detection of left atrial enlargement. Correlation of $P$ wave with left atrial dimension by echocardiography. Heart [Internet]. 1975 Dec 1;37(12):1281-5. Available from: http://heart.bmj. com/content/37/12/1281.abstract

9. Dixen $U$, Vang Larsen $M$, Ravn L, Parner J, Jensen GB. Signal-averaged $P$ wave duration and the long-term risk of permanent atrial fibrillation. Scand Cardiovasc J [Internet]. 2008 Jan 12;42(1):31-7. Available from: https://doi. org/10.1080/14017430701652282

10. Platonov PG. P-Wave Morphology: Underlying Mechanisms and Clinical Implications. Ann Noninvasive Electrocardiol [Internet]. 2012 Jul;17(3):161-9. Available from: http://doi.wiley.com/10.1111/i.1542-474X.2012.00534.X

11. Baranchuk A, Bayés de Luna A. The P-wave morphology: what does it tell us? Herzschrittmachertherapie + Elektrophysiologie [Internet]. 2015 Sep 12;26(3):192-9. Available from: http://link.springer.com/10.1007/s00399-015-0385-3

12. D’Andrea A, De Corato G, Scarafile R, Romano S, Reigler L, Mita C, et al. Left atrial myocardial function in either physiological or pathological left ventricular hypertrophy: a two-dimensional speckle strain study. Br J Sports Med [Internet]. 2008 Aug;42(8):696-702. Available from: http://www.ncbi.nlm.nih.gov/pubmed/18070810

13. Wijffels MC, Kirchhof CJ, Dorland R, Allessie MA. Atrial fibrillation begets atrial fibrillation. A study in awake chronically instrumented goats. Circulation [Internet]. 1995 Oct 1;92(7):1954-68. Available from: http://www.ncbi.nlm.nih.gov/pu$\underline{\mathrm{bmed} / 7671380}$

14. Sato T, Mitamura H, Kurita Y, Takeshita A, Shinagawa K, Miyoshi S, et al. Recovery of electrophysiological parameters after conversion of atrial fibrillation. Int J Cardiol [Internet]. $2001 \mathrm{Jul} ; 79(2-3): 183-9$. Available from: http://www.ncbi.nlm.nih. gov/pubmed/11461740

15. Dilaveris P, Batchvarov V, Gialafos J, Malik M. Comparison of different methods for manual P wave duration measurement in 12-lead electrocardiograms. Pacing Clin Electrophysiol [Internet]. 1999 Oct;22(10):1532-8. Available from: http://www.ncbi.nlm.nih.gov/pubmed/10588156

16. Dilaveris PE, Gialafos JE. P-wave dispersion: a novel predictor of paroxysmal atrial fibrillation. Ann Noninvasive Electrocardiol [Internet]. 2001 Apr;6(2):159-65. Available from: http://www.ncbi.nlm.nih.gov/pubmed/11333174

17. Zimmer K, Przywara W, Zyśko D, Sławuta A, Gajek J. The nature of P-wave dispersion - A clinically useful parameter that does not exist. Int J Cardiol [Internet]. 2016 Jun 1;212:59-60. Available from: http://www.ncbi.nlm.nih.gov/pubmed/27031821

18. Bayés de Luna A, Baranchuk A, Alberto Escobar Robledo L, Massó van Roessel A, Martínez-Sellés M. Diagnosis of interatrial block. J Geriatr Cardiol [Internet]. 2017 Mar;14(3):161-5. Available from: http://www.ncbi.nlm.nih.gov/pubmed/28592957

19. Bayés de Luna A, Platonov P, Cosio FG, Cygankiewicz I, Pastore C, Baranowski R, et al. Interatrial blocks. A separate entity from left atrial enlargement: a consensus report. J Electrocardiol [Internet]. 2012 Sep;45(5):445-51. Available from: http://www.ncbi.nlm.nih.gov/pubmed/22920783

20. Bagliani G, Michelucci A, Angeli F, Meniconi L. Atrial activation analysis by surface P wave and multipolar esophageal recording after cardioversion of persistent atrial fibrillation. Pacing Clin Electrophysiol [Internet]. 2003 May;26(5):1178-88. Available from: http://www.ncbi.nlm.nih.gov/pubmed/12765445

21. Paslawska U, Noszczyk-Nowak A, Paslawski R, Janiszewski A, Kiczak L, Zysko D, et al. Normal electrocardiographic and echocardiographic (M-mode and two-dimensional) values in Polish Landrace pigs. Acta Vet Scand [Internet]. 2014 Sep 9;56:54. Available from: http://www.ncbi.nlm.nih.gov/pubmed/25196530 